\title{
Erratum: Transverse momentum broadening from the lattice [Phys. Rev. D 101, 014505 (2020)]
}

\author{
Guy D. Moore and Niels Schlusser@
}

(Received 5 March 2020; accepted 9 March 2020; published 20 March 2020)

DOI: 10.1103/PhysRevD.101.059903

We recently calculated the soft contribution to $C\left(\boldsymbol{b}_{\perp}\right)$ nonperturbatively on the lattice in our paper. Due to a mistake in our fitting procedure, our results were off by a factor of 2 . We correct that mistake and discuss how the corrected results match our expectations.

Computational details.-In our paper we investigated the length dependence of the log determinant of a modified Wilson loop of length $L$. Unfortunately, in our fitting procedure we incorrectly treated the length of the loops; specifically, in Eq. (3.3) we had

$$
\left(g_{3 \mathrm{~d}}^{2} L\right)_{\text {actual }}=1.0+\left(\frac{g_{3 \mathrm{~d}}^{2} L}{2}\right)_{\text {assumed }} .
$$

Therefore the extracted value of $(d / d L)-\ln \operatorname{Tr} W(L)$ and the impact parameter-space scattering kernel $C\left(\boldsymbol{b}_{\perp}\right)$ are incorrectly evaluated by precisely a factor of $1 / 2$, while the (irrelevant) prefactors $c_{i}$ receive an additional factor of

$$
\left(c_{i}\right)_{\text {actual }}=\mathrm{e}^{-C\left(\boldsymbol{b}_{\perp}\right) / g_{3 \mathrm{~d}}^{2}}\left(c_{i}\right)_{\text {assumed }} .
$$

Correcting this error revises our results for $C\left(\boldsymbol{b}_{\perp}\right)$ : Table II should be replaced by Table I. We also provide updated figures in Figs. 1 and 2.

TABLE I. Results for $C\left(\boldsymbol{b}_{\perp}\right)$ for four temperatures and a range of transverse separations. All data points are continuum extrapolated, with errors representing all statistical and systematic errors associated with the data extraction and extrapolations, except for the first (smallest $g_{3 \mathrm{~d}}^{2} b_{\perp}$ ) entry in each column, which is based on one lattice spacing. We also quote the extracted value of $\hat{q}$ and the string

\begin{tabular}{|c|c|c|c|c|}
\hline$\underline{g_{3 \mathrm{~d}}^{2} b_{\perp}}$ & $\begin{array}{l}x_{\text {cont }}=0.08896 \\
y_{\text {cont }}=0.452423 \\
\frac{C\left(\boldsymbol{b}_{\perp}\right)}{g_{3 \mathrm{~d}}^{\perp}} \mid \begin{array}{l}N_{\mathrm{f}}=3 \\
250 \mathrm{MeV}\end{array}\end{array}$ & $\begin{array}{l}x_{\text {cont }}=0.0677528 \\
y_{\text {cont }}=0.586204 \\
\left.\frac{C\left(\boldsymbol{b}_{\perp}\right)}{g_{3 \mathrm{~d}}^{2}}\right|_{500 \mathrm{MeV}} ^{N_{\mathrm{f}}=3} \\
\end{array}$ & $\begin{array}{l}x_{\text {cont }}=0.0463597 \\
y_{\text {cont }}=0.823449 \\
\left.\frac{C\left(\boldsymbol{b}_{\perp}\right)}{g_{3 \mathrm{~d}}^{2}}\right|_{1 \mathrm{GeV}} ^{N_{\mathrm{f}}=4} \\
\end{array}$ & $\begin{array}{l}x_{\text {cont }}=0.0178626 \\
y_{\text {cont }}=1.64668 \\
\left.\frac{C\left(\boldsymbol{b}_{\perp}\right)}{g_{3 \mathrm{~d}}^{2}}\right|_{100 \mathrm{GeV}} ^{N_{\mathrm{f}}=5} \\
\end{array}$ \\
\hline 0.125 & $-0.0058(44)$ & & & $-0.005(19)$ \\
\hline 0.25 & $-0.0091(36)$ & $-0.0130(36)$ & $-0.0094(34)$ & $0.003(32)$ \\
\hline 0.5 & $-0.01154(63)$ & $-0.00394(87)$ & $0.00599(58)$ & $0.03166(49)$ \\
\hline 0.75 & $0.0000(11)$ & $0.0128(17)$ & $0.0337(10)$ & $0.07904(81)$ \\
\hline 1.0 & $0.00623(82)$ & $0.03313(61)$ & $0.06366(36)$ & $0.12649(33)$ \\
\hline 1.5 & $0.0606(17)$ & $0.1055(11)$ & $0.15803(94)$ & $0.25599(62)$ \\
\hline 2.0 & $0.1269(33)$ & $0.2002(28)$ & $0.2712(18)$ & $0.3986(12)$ \\
\hline 2.5 & $0.2150(41)$ & $0.3009(51)$ & $0.3947(32)$ & $0.5518(22)$ \\
\hline 3.0 & $0.3114(86)$ & $0.4164(46)$ & $0.5234(67)$ & $0.7048(45)$ \\
\hline 4.0 & $0.435(42)$ & $0.643(33)$ & $0.790(28)$ & $1.053(17)$ \\
\hline 5.0 & $0.726(94)$ & $0.941(96)$ & $1.02(10)$ & $1.314(13)$ \\
\hline 6.0 & $1.21(11)$ & $1.35(10)$ & $1.60(15)$ & $1.700(36)$ \\
\hline$\hat{q} / g_{3 \mathrm{~d}}^{6}$ & $0.1847(78)$ & $0.230(10)$ & $0.3637(60)$ & $0.6424(47)$ \\
\hline$\sigma_{\mathrm{EQCD}} / g_{3 \mathrm{~d}}^{4}$ & $0.2836(10)$ & $0.2867(10)$ & $0.2901(11)$ & $0.2952(11)$ \\
\hline
\end{tabular}
tension as determined in [1], see text.

Published by the American Physical Society under the terms of the Creative Commons Attribution 4.0 International license. Further distribution of this work must maintain attribution to the author(s) and the published articles title, journal citation, and DOI. 


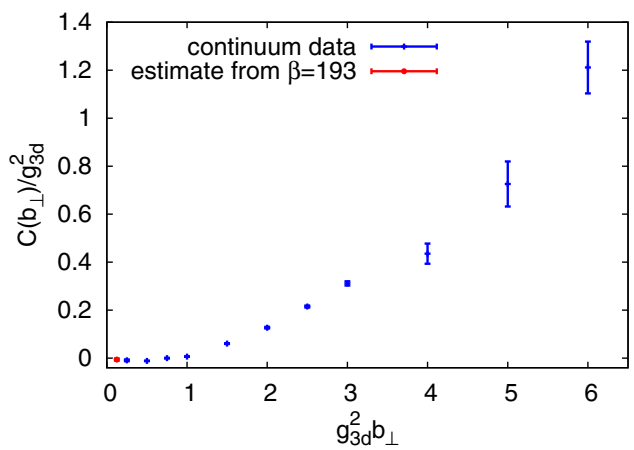

(a) $x=0.08896$

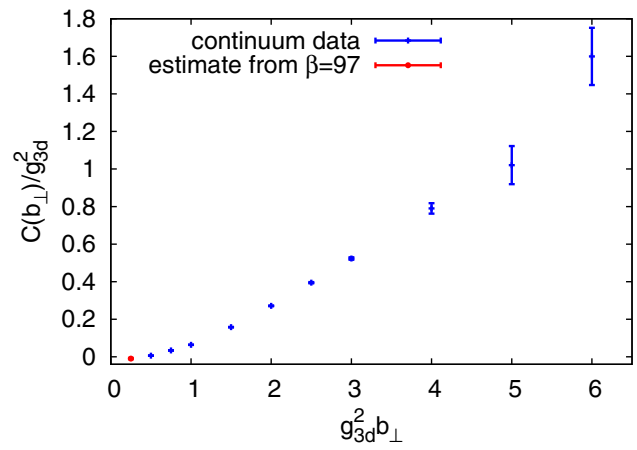

(c) $x=0.0463597$

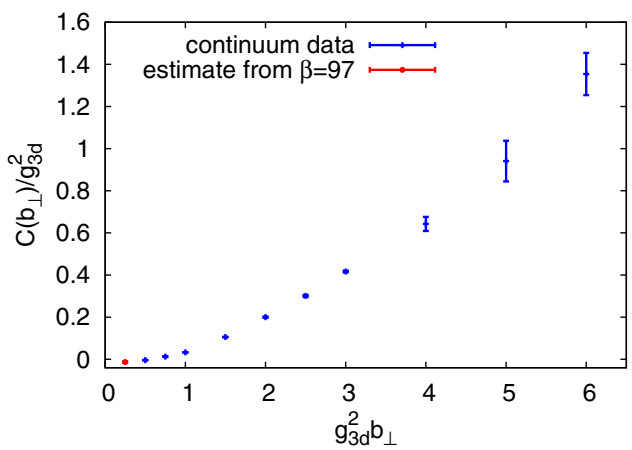

(b) $x=0.0677528$

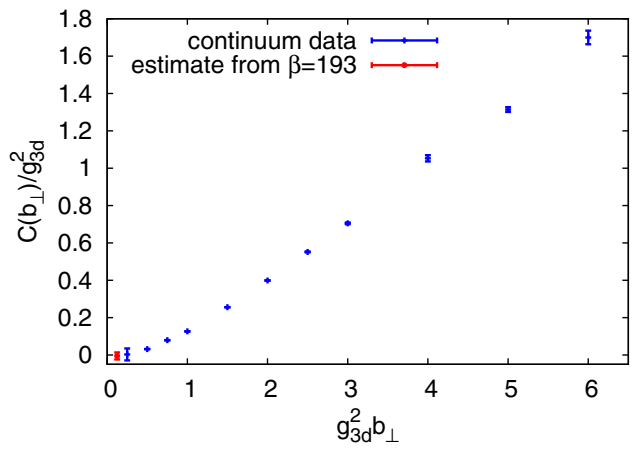

(d) $x=0.0178626$

FIG. 1. $C\left(\boldsymbol{b}_{\perp}\right)$ for different $x$. The blue points are based on continuum extrapolations; the red points are from a single lattice spacing, as described in the text.
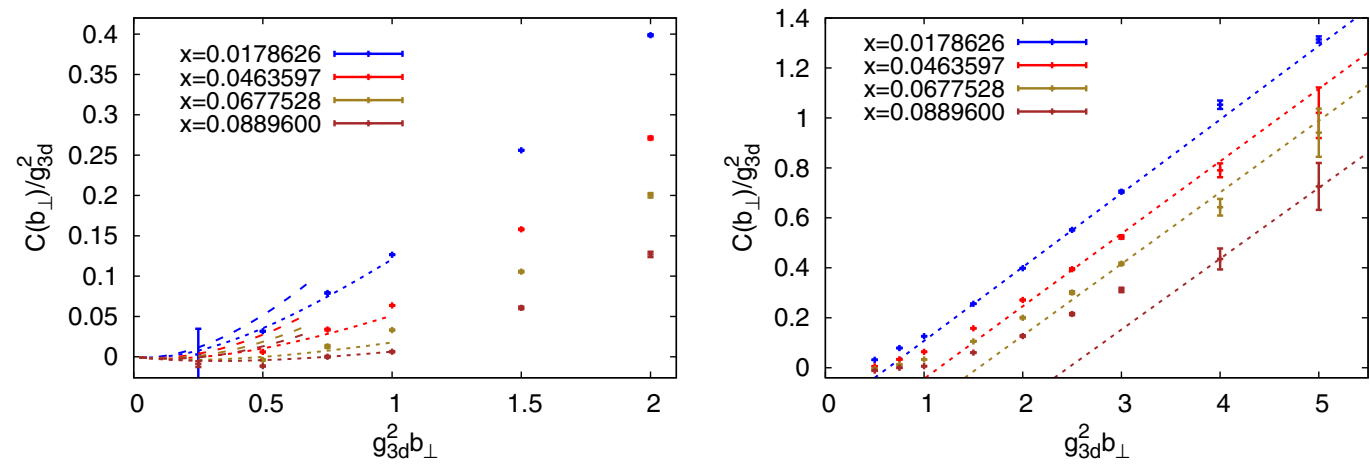

FIG. 2. Left: $C\left(\boldsymbol{b}_{\perp}\right)$ for four temperatures at small $g_{3 \mathrm{~d}}^{2} b_{\perp}$, with linear-plus-quadratic fits (dotted) and linear-plus-quadratic NLO perturbative predictions (dashed). Right: large- $b_{\perp}$ behavior of $C\left(\boldsymbol{b}_{\perp}\right)$, with linear expectations based on the EQCD string tension.

The data in the appendixes remains unchanged since constant factors do not influence the correlation matrix, only the error and covariance matrix.

Discussion. Analytical expectations: Large distance.-As already emphasized in the original paper, at large $b_{\perp}$ the scalar field has almost vanishing correlations between the two sides of the modified Wilson loop, so its $b_{\perp}$ dependence arises from gauge fields and should show the same (area-law) large $b_{\perp}$ behavior as an ordinary Wilson loop, which is set by the string tension in EQCD. The EQCD string tension was predicted in [1], by performing a matching between EQCD and 3D pure-glue QCD (MQCD) due to [2] and the MQCD string tension results of [3]. The prediction is

$$
\frac{\sqrt{\sigma_{\mathrm{EQCD}}}}{g_{3 \mathrm{~d}}^{2}}=\left[1-\frac{1}{48} \frac{3}{\pi \sqrt{y}}-\frac{17}{4608}\left(\frac{3}{\pi \sqrt{y}}\right)^{2}\right] \times 0.553(1)
$$


which now agrees well with the large $b_{\perp}$ region of our data, as shown in the right panel of Fig. 2 . The smaller- $x$ data has a heavier scalar field and the convergence to the EQCD behavior is therefore expected to occur sooner, exactly as we find.

Discussion. Value of $q$-hat.-We repeat our extraction of $\hat{q}$ from the small- $b_{\perp}$ data; the result is shown in the second-tolast line of the table. For the smallest three temperatures (largest $x$ values), $\hat{q}$ is still about a factor of 2 smaller than the NLO estimate, indicating that in these cases the next correction is not small. However, the deviation at the largest temperature, where perturbation theory is expected to work better, is only a factor of 1.3 , indicating that the perturbative expansion is becoming more reliable.

[1] M. Laine and Y. Schroder, Two-loop QCD gauge coupling at high temperatures, J. High Energy Phys. 03 (2005) 067.

[2] P. Giovannangeli, Two loop renormalization of the magnetic coupling in hot QCD, Phys. Lett. B 585, 144 (2004).

[3] M. J. Teper, SU(N) gauge theories in (2+1)-dimensions, Phys. Rev. D 59, 014512 (1998). 